Japanese Psychological Research

1987. Vol. 29, No. 4, 173-183

\title{
Estimated duration for rotating-spot-pattern
}

\author{
T.ADAYUKI T.AYAMA, MASATO NAKAMURA \\ and THOMAS S. AIBA' \\ Department of Beluwioral Science, Faculty of Letters, Hokkaido University, Kita-ku, Sapporo 060
}

\begin{abstract}
Two experiments were carried out to investigate duration estimation of rotating-spot-patterns under a situation with the minimum cue to frequency. Twenty-four undergraduates were used as subjects in each experiment. The magnitude estimation method was used in Experiment I, where for a constant duration, the estimate of the duration increased with the velocity of the rotation of the pattern except for that of stationary pattern, where the duration was estimated longer than that for the slowest of the moving patterns. The same tendencies were observed in Experiment II, where the magnitude production method was used. The multiple regression analysis suggested that the best model which explains the velocity effects upon time estimation was a multiplicative one and contained a quadratic function of logarithmically transformed velocity in both experiments. The explanatory principles of the velocity effects and the validity of the model were discussed in the light of past studies.
\end{abstract}

Key words: time estimation, duration, velocity, rotating-spot-pattern, magnitude estimation, magnitude production.

There have been several studies investigating duration estimation related to movement (Fraisse, 1962; Lhamon \& Goldstone, 1975; Matsuda, 1974; Rachlin, 1966; Tayama \& Aiba, 1982; Tayama, 1986). Two main effects have been uncovered in these studies, both related to the spatial and temporal attributes of the stimulus. One of them is the effect of velocity (or of distance) upon duration estimation. The other is the difference between estimated durations for a moving stimulus and for a stationary stimulus.

Fraisse (1962) found that the reproduced durations for higher velocity movement judged against standard durations of a lower velocity movement are longer than the reverse. Rachlin (1966) also showed that with real durations held constant, the estimated durations for a fast moving spot appeared longer than that for a slower one. On the other hand, Matsuda (1974) found that even though

1 The authors wish to thank Dr. Kazuo Mori of College of Education, Shinshu University, for his reading and helpful comments on the first manuscripts. the physical durations of a standard stimulus were constant, reproduced durations for a higher velocity standard (or for a standard of longer distance) were found to be shorter than that of lower velocity (or of shorter distance). Since in these experiments, a single moving stimulus was used and the velocity of the stimulus was always interrelated with the length of its moving path, it was not possible to decide which of them, velocity or distance, caused the difference in duration estimation. In the classical studies, Abbe (1935) and Cohen, Hunsel, and Sylvester (1955) found that the apparent duration was longer when the distance between stimuli was larger. On the other hand, Tayama and Aiba (1982), using random-spot-patterns as stimuli, found the effect of velocity where effects of distance were eliminated; the estimated duration increased with the increase of velocity. From what we have seen, the estimated duration seems to be affected by both velocity and distance.

With respect to the comparison of duration estimations for moving vs. stationary stimuli, Lhamon and Goldstone 
(1975) reported that a moving horizontal grating pattern was judged longer in time than the same pattern which was stationary. Tayama and Aiba (1982) also found that the duration of the moving pattern was usually estimated longer than that of the stationary pattern. From the studies of velocity effects, it should be expected that the duration of moving stimuli is always estimated longer than that of stationary stimuli or the reverse. But it was not the case in the Tayama and Aiba experiment. The estimate for stationary pattern was usually smaller than that for a moving pattern but larger than that for a slowly moving pattern.

There have been found other effects of stimulus attributes upon the duration estimation, such as numerosity (Mo, 1971), size (Thomas \& Cantor, 1976), complexity (Ornstein, 1969), frequency (Thomas \& Brown, 1974), and familiarity (Avant, Lyman, \& Antes, 1975). Some ingenious investigators have proposed some models and hypotheses to account for their effects (Fraisse, 1963; Ornstein, 1969; Thomas \& Weaver, 1975; Block, 1978, 1979). But such models and hypotheses do not fit completely to the movement effects mentioned above.

The present study chiefly aims at confirming Tayama and Aiba's findings through experiments which differed from theirs and from some others in two important aspects meanwhile proposing a model which would fit results well. First, Tayama and Aiba used as stimuli random-spot-patterns moving horizontally. But using such stimuli, the factor of stimulus frequency might have been present as some kind of cue. Thus in the present experiments another kind of stimuli, rotating-spot-patterns in which the number of spots always remained constant, were used to eliminate the frequency factor and to examine what would be the pure effects of velocity. Secondly, the two effects already mentioned, the velocity effect and the difference between durations for the moving pattern and for the stationary pattern, have been mostly found in verbal estimation method. Thus to examine whether two effects had not been due to artifacts inherent in such a particular experimental method, two methods were used and results compared; magnitude estimation (Experiment I) and magnitude production (Experiment II). Through comparison and detailed analysis of the two sets of experimental data, we will seek for a best explanatory model which explains the data well.

\section{Experiment I}

\section{Method}

Subjects. Twenty-four undergraduates of Hokkaido University served as subjects; there were sixteen males and eight females, all of whom had normal visual acuity either uncorrected or corrected by spectacle lenses.

Stimuli and apparatus. The rotating pattern used (Tayama, 1984) consisted of sixteen illuminated spots each of which was located on points of intersection between each of eight lines radiating from a pivotal point at equal angles of $45 \mathrm{deg}$ and each of two concentric circles centered around the same point (diameters

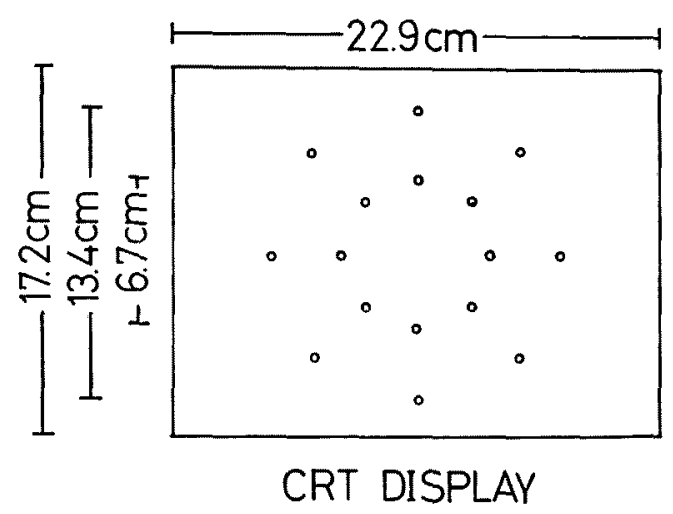

Fig. 1. Stimulus pattern composed of sixteen luminous spots which form two concentric circles. 
are $6.7 \mathrm{~cm}$ and $13.4 \mathrm{~cm}$ ) (Fig. 1). This pattern rotates clockwise around the point already mentioned. A mini-computer (DEC PDP-8/e) was used to control the experiment. The stimuli were presented on a fast-decay display (DEC VR 14) under the control of the computer. The stimulus luminance was constant at about $800 \mathrm{~cd} / \mathrm{m}^{2}$. In front of the display, there were a chair and a chin and head-rest to keep the subject's face in position. The distance between the display and subject's eye was $90 \mathrm{~cm}$. A microphone was set in front of his mouth. An amplifier amplified voice sounds and an A-D converter changed them into digital signals. The computer fetched the signals if the sound voltages exceeded a preset level. Once a signal was received by the computer, 2 s later, a bell of the computerkeyboard rang to make subjects ready for the next trial and after the interval of $2 \mathrm{~s}$ the stimuli were presented, and this cycle was repeated automatically. The internalclock of the computer was used as a time controller of stimulus presentations. The experiment was carried out in a darkened room.

Procedure. Each subject was instructed to observe the stimulus pattern binocularly without particular restraint and to estimate the duration between the onset and offset of the pattern by the method of magnitude estimation, that is, to assign an appropriate number verbally to each comparison pattern, relative to the standard pattern (the modulus being given the magnitude of 100). The standard pattern rotates at the angular velocity of 1.2 $\mathrm{rad} / \mathrm{s}$ for $6 \mathrm{~s}$. For the comparison pattern there were four velocity conditions $(0,0.3$, 1.2 , and $2.4 \mathrm{rad} / \mathrm{s}$ ) and six duration conditions $(2,4,6,9,12$, and $16 \mathrm{~s})$, yielding $4 \times 6$ different conditions. The standard pattern and the following 24 randomly ordered comparison patterns formed a stimulus session. Each subject had a practice of ten trials beforehand and then made observations and judgments in four different stimulus sessions and between the sessions a few minutes rest was inserted. The total trial number was 106. The responses were recorded by the experimenter, listening to the subject in the adjoining room via a microphone and a speaker. Each subject took about one hour to complete the task for this experiment.

\section{Results and Discussion}

The mean values of magnitude estimation of each condition in each subject were calculated. An analysis of variance was performed on the estimation values with respect to the variables of Stimulus Duration, Velocity and Subject. We obtained significant main effects of Duration $(F(5, \quad 115)=215.26, \quad p<.001)$ and Velocity $(F(3,69)=32.75, p<.001)$ and the significant interaction between them $(F(15,345)=5.71, \quad p<.01)$. The main effects were also significant when the interaction was used as the error term $(F(5,15)=37.70, p<.001 ; F(3,15)=5.74$, $p<.01)$. The partial correlation coefficients, $r_{e v \cdot t}$ and $r_{e t \cdot v}$, where $e, v$ and $t$ indicate the estimation value, the stimulus velocity and the stimulus duration, were

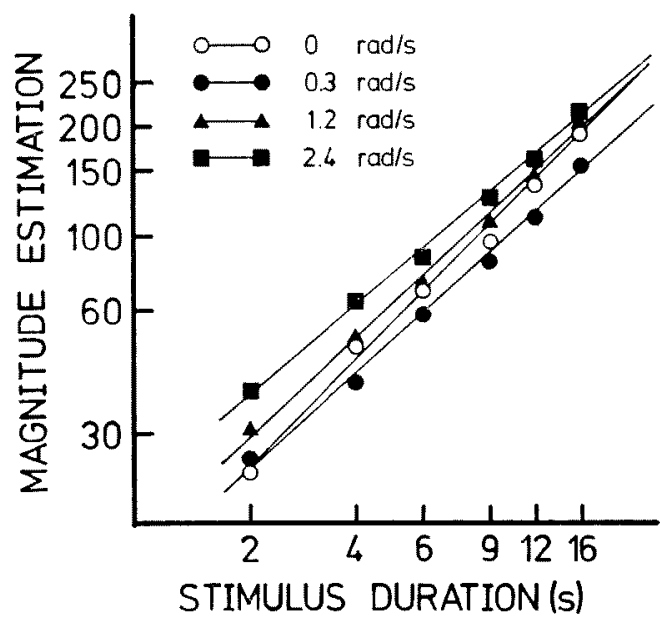

Fig. 2. Numerical estimates for rotating-dotpattern as a function of actual duration. The parameter is the velocity at which the pattern rotates. The modulus $(6 \mathrm{~s}, 1.2 \mathrm{rad} / \mathrm{s})$ was called 100 . The exponent for all curves was about 0.9 . 
0.197 and 0.764 in that order.

The mean estimated values in each condition are plotted logarithmically in Fig. 2. This figure shows that, though the stimulus durations of comparison pattern were equal, the estimated durations increased with the rotation velocity except in the condition of $0 \mathrm{rad} / \mathrm{s}$ (stationary). The estimated values were divided by the stimulus durations and the differences in the mean values between the velocity conditions were examined by multiple comparison method (Turkey's method). The means of $2.4 \mathrm{rad} / \mathrm{s}$ was significantly larger than those of $1.2 \mathrm{rad} / \mathrm{s}$ which was also significantly larger than $0 \mathrm{rad} / \mathrm{s}(q=$ $4.6, W S D=5.02, p<.01)$. The means of $0.3 \mathrm{rad} / \mathrm{s}$ was significantly smaller than $0 \mathrm{rad} / \mathrm{s}$. Clearly, the estimates of duration increased with the velocity of moving pattern, except for the stationary pattern.

The exponents of power function, for the plots of the estimated values against the stimulus durations, calculated by least square method for each velocity condition, were $0.99,0.91,0.96$, and 0.84 , respecting for $0,0.3,1.2$, and $2.4 \mathrm{rad} / \mathrm{s}$ and totally was .92 . The exponent (.99) for the stationary pattern $(0 \mathrm{rad} / \mathrm{s})$ was higher than that for the moving pattern. Within the moving patterns, the exponent decreased with velocity of the patterns.

As pointed out, the estimates of duration increased with the velocity of moving pattern, except that the estimates for stationary pattern $(0 \mathrm{rad} / \mathrm{s})$ were not necessarily smaller than those for moving pattern. In the next experiment, we will test if the method (magnitude production) other than those previously used by other investigators would still produce the same effects.

\section{Experiment II}

\section{Method}

Subjects. Twenty-four undergraduates of Hokkaido University served as sub- jects; there were seventeen males and seven females, half of whom had also served in Experiment I.

Stimuli and apparatus. The same patterns as those used on Experiment I was used as stimuli. The apparatus were also almost the same as in Experiment I, but, the microphone, the amplifier and the A-D converter used as a voice key were not used. Instead, a keyboard was put beside the subject as a response key. A computer program was written to repeat the following sequence; once the key of the keyboard was pushed, the pattern was presented and at the same time the clock began to count the time marker. When the key was pushed again, the pattern disappeared and the clock stopped to record the produced time. The other things were equal to Experiment I.

Procedure. At first each subject was presented a standard stimulus whose velocity was $1.2 \mathrm{rad} / \mathrm{s}$ for $6 \mathrm{~s}$ which was the same as in Experiment I. The number 100 was assigned to the duration of the standard stimulus. Then, he or she was given in succession a number by an experimenter, and was asked to produce a duration corresponding to the given number by pushing the key twice, an interval between them being the produced duration. The numbers given by the experimenter were $30,60,100$, 150,200 , and 250 (proportionally corresponding to $1.8,3.6,6,9,12$, and $15 \mathrm{~s}$ ). There were four levels to the velocity of the pattern, i.e., $0,0.3,1.2$, and $2.4 \mathrm{rad} / \mathrm{s}$ which were the same as in Experiment $I$. This yielded $6 \times 4$ combinations. The standard stimulus and the following 24 randomly ordered stimuli constitutedstimulus session. The other things were equal to Experiment $\mathrm{I}$.

\section{Results and Discussion}

The mean produced durations of each condition in each subject were calculated. An analysis of variance was performed with respect to Durations with Assigned 
Number, Stimulus Velocity and Subject as variables. We found significant main effects of Assigned Number $(F(5,115)=$ $165.33, p<.001)$ and Stimulus Velocity $(F(3,69)=25.30, p<.001)$. The interaction between them was also significant $(F(15,3+5)=2.89, \quad p<.01)$. The main eflects when the interaction was used as the error term were also significant $(F(5,15)=57.21, p<.001 ; F(3,15)=8.75$, $p<.01)$. The partial correlation coefficients, $r_{t v \cdot n}$ and $r_{t n \cdot t}$, where $t, v$ and $n$ indicate the produced duration, the stimulus velocity and the assign number, were -0.156 and 0.629 in that order.

The mean produced durations in each condition are plotted logarithmically in Fig. 3. It should be noted that in this figure the ordinate indicates the assigned number and the abscissa the produced duration. The differences between the velocity conditions in the mean produced durations pooled with respect to the assign numbers were examined by the multiple comparison method. Between any pairs of mean produced durations in the four velocity conditions, there was a significant difference $(q=4.6, W S D=0.57$,

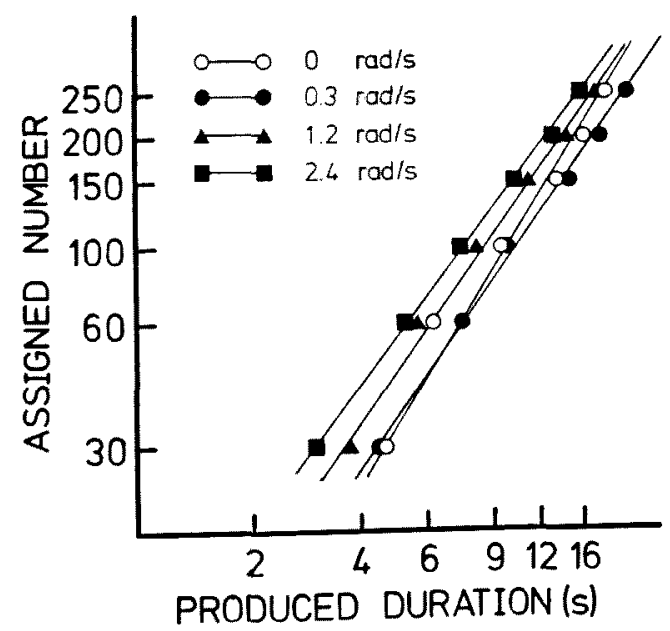

Fig. 3. Subjective magnitude in assigned numbers as a function of actually produced duration. The parameter is the velocity. The number 100 was assigned to the standard stimulus $(6 \mathrm{~s}, 1.2 \mathrm{rad} /$ s). The exponent for all curves was about 1.25. $p<.01)$. The produced durations for moving patterns decreased with velocity even though the assigned numbers were the same. Moreover the produced durations for the stationary pattern $(0 \mathrm{rad} / \mathrm{s})$ were shorter than those for $0.3 \mathrm{rad} / \mathrm{s}$ and longer than for 1.2 and $2.4 \mathrm{rad} / \mathrm{s}$. These were results almost symmetrical to the estimated values in Experiment I. But if we examine the subject's estimation against the stimulus duration, we could consider the assigned number in Experiment II to correspond to the estimated value in Experiment $I$ and, likewise the produced duration to the stimulus duration. From this point of view, as already seen with Experiment I, the subjects estimated the duration longer for the higher velocity patterns than that for the lower ones. Again, the durations for stationary pattern were not necessarily estimated shorter than those for moving patterns.

The exponents of power function, in plots of the assigned number vs. the produced duration, were $1.319,1.277,1.235$, and 1.183 in $0,0.3,1.2$, and $2.4 \mathrm{rad} / \mathrm{s} \mathrm{con-}$ ditions respectively, and totally 1.252 .

\section{Comparison between Experiments I and II}

In the following analysis we will directly compare estimated values vs. stimulus durations in Experiment $I$ and assign numbers vs. mean produced durations in Experiment II.

\section{Estimated One Second}

To compare the velocity effects in both experiments, the estimated-one-seconds (EOS in abbreviated form) were computed in each velocity condition. EOS in Experiment I was given by the following equation,

$$
E O S=(m d \times E N) /(s d \times M O D)
$$

where $s d$ is the stimulus duration, $E N$ the estimated value (number) for $s d, M O D$ 
Table 1

The comparison of estimated-one-seconds between Experiment I and Experiment II

\begin{tabular}{ccc}
\hline $\begin{array}{c}\text { Velocity } \\
\text { conditions } \\
\text { (rad/s) }\end{array}$ & $\begin{array}{c}\text { Experiment I } \\
(\mathrm{s})\end{array}$ & $\begin{array}{c}\text { Experiment II } \\
(\mathrm{s})\end{array}$ \\
\hline 0 & 0.698 & 0.637 \\
0.3 & 0.625 & 0.581 \\
1.2 & 0.759 & 0.700 \\
2.4 & 0.895 & 0.768 \\
\hline Mean & 0.744 & 0.672 \\
\hline
\end{tabular}

the modulus (100 in Experiment I) and $m d$ the duration of the standard (6s in $\mathrm{Ex}$ periment I). EOS's in Experiment II was also calculated by the following equation,

$$
E O S=(m d \times A \mathcal{N}) /(p d \times M O D)
$$

where $A J$ is the number assigned by experimenter, and $p d$ the produced duration corresponding to $A \mathcal{N}$. The value of $M O D$ and $m d$ were the same as Experiment I. The results of mean EOS's are given in Table 1. This table shows that the results of Experiment I were very similar to those of Experiment II. The difference in the estimates between the two experiments is smaller than that among velocity conditions. To recapitulate the similarity between the experiments: (i) the stimulus duration $1 \mathrm{~s}$ was underestimated, (ii) the higher was the velocity for the moving pattern, the larger was the estimate, and (iii) the estimate for the slowest velocity condition (stationary pattern) was not the smallest.

\section{Exponents}

As for the exponents, we obtained, on one hand, a difference in the absolute exponent values between the two experiments. Figure 4 represents this difference. In this figure the mean estimated values in each duration condition (Experiment I) and the mean produced durations in each assigned number (Experiment II) are both plotted. The difference of the slopes of the two lines reflected the absolute difference of exponent values, the slope for the Experiment II being steeper than that for Experiment I. This seems to result from the central tendency or regression effect in which subjects took values ("numbers" in Experiment $I$ and "durations" in Experiment II) toward the middle value of the whole memorized range. Namely, this difference can be attributed to the difference of methods in both experiments. On the other hand we obtained basically the same results in both experiments; the values of the exponent increased with the decrease of velocity for the pattern including that of $0 \mathrm{rad} / \mathrm{s}$. This suggests that under the lower velocity conditions the subjects estimated the duration longer in the range of long durations and estimated shorter in the range of short durations in comparison to his judgment under the higher velocity conditions, and this differences of duration estimation between velocity conditions are consistent irrespective of the difference of methods.

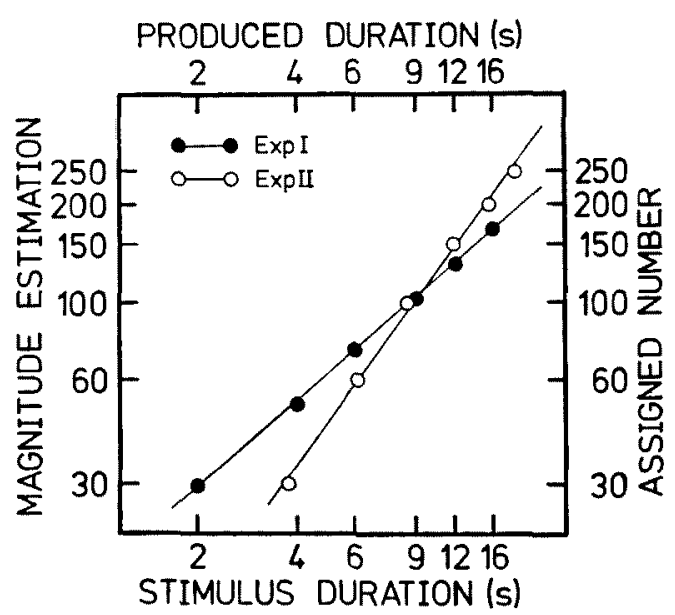

Fig. 4. The comparison of mean estimated durations between Experiment I (magnitude estimation) and Experiment II (magnitude production). Filled circles: average estimates in each duration condition of Experiment I, empty circles: average produced durations in each assigned number condition of Experiment II. 
Table 2

The results of multiple regression analysis

\begin{tabular}{|c|c|c|c|c|c|c|c|}
\hline \multirow{2}{*}{ No. } & \multirow{2}{*}{ Models } & \multicolumn{3}{|c|}{ Experiment I } & \multicolumn{3}{|c|}{ Experiment II } \\
\hline & & Parameter estimates & $R^{2}$ & AlC & Parameter estimates & $R^{4}$ & $\mathrm{AIC}$ \\
\hline (1) & $E=a+b T+c T^{\prime}$ & $\begin{aligned} E & =-.104+.649 T \\
& +.597 \mathrm{~V}\end{aligned}$ & .979 & -30.7 & $\begin{aligned} E= & -2.807+.893 T \\
& +.943 V\end{aligned}$ & .978 & -12.1 \\
\hline (2) & $E=a+b T$ & $E=4.258+.191 V T$ & .359 & 48.9 & $E=6.877+.057 \mathrm{VT}$ & .107 & 74.7 \\
\hline (3) & $\begin{aligned} E & =a+b T+c V^{*} \\
& +d I^{\circ}\end{aligned}$ & $\begin{aligned} E & =.007+.649 T \\
& +.202 V^{2}+.015 V\end{aligned}$ & .980 & -30.3 & $\begin{aligned} E= & -2.787+.809 T \\
& +.038 V^{2}+851 V\end{aligned}$ & .978 & -10.1 \\
\hline (4) & $\begin{aligned} E & =a+T\left(b \Gamma^{\circ}\right. \\
& \left.+c \Gamma^{+}+d\right)\end{aligned}$ & $\begin{aligned} E= & .478+T(.0811 \\
& +.015 V+0.60 \mathrm{I})\end{aligned}$ & .976 & -25.7 & $\begin{aligned} E= & -1.891+T\left(.011 V^{2}\right. \\
& +.059 V+0.823)\end{aligned}$ & .981 & -14.9 \\
\hline (5) & $\begin{aligned} E & =a+b T \\
& +c \log V\end{aligned}$ & $\begin{aligned} E & =.617+.649 T \\
& +.182 \log V\end{aligned}$ & .954 & -12.9 & $\begin{aligned} E= & -1.372+.870 \tau \\
& +.335 \log V\end{aligned}$ & .954 & -12.9 \\
\hline (6) & $E=a+b T \log V$ & $\begin{aligned} E & =5.453 \\
& -.052 T \log r\end{aligned}$ & .005 & 58.5 & $\begin{aligned} E & =7.315 \\
& -.065 T \log V\end{aligned}$ & .056 & 76.0 \\
\hline (7) & $\begin{array}{l}E=a+b T \\
\quad+c\left(\log T^{2}\right. \\
\quad+d \log T^{2}\end{array}$ & $\begin{aligned} E & =.477+.649 T \\
& +.682(\log V)^{2} \\
& +2.097 \log V\end{aligned}$ & .989 & -45.7 & $\begin{aligned} E & =-1.943+.904 T \\
& +1.025(\log V)^{2} \\
& +3.224 \log V\end{aligned}$ & .989 & -26.6 \\
\hline (8) & $\begin{array}{l}E=a \\
\quad+T\left(b(\log V)^{2}\right. \\
+c \log V+d)\end{array}$ & $\begin{aligned} E & =.478 \\
& +T\left(.075(\log V)^{2}\right. \\
& +.225 \log V+.645)\end{aligned}$ & .990 & -46.7 & $\begin{aligned} E & =-2.017 \\
& +T\left(.089(\log V)^{2}\right. \\
& +.281 \log V+.920)\end{aligned}$ & .994 & -40.9 \\
\hline
\end{tabular}

Note. $E, T$ and $V$ indicate the estimated value, the stimulus (produced) duration and the stimulus velocity and $a, b, c$ and $d$ are parameters.

\section{Multiple Regression Analysis}

Next, we will examine and compare the direct relations and interactions among three variables, i.e., duration, velocity and the estimated value by multiple regression analysis and make clear the influence of velocity upon time estimation in both experiments. We can simply think of two kinds of model which are appropriate to represent the relationship. That is,

$$
\begin{aligned}
& E(T, V)=F(\mathcal{T})+G(V) \\
& E(T, V)=F(\mathcal{T}) \times G(V)
\end{aligned}
$$

Here $E(T, V)$ is the estimated value. $F(T)$ and $G(V)$ represent, respectively the function of time and the function of velocity. The model (3) means that the estimated values are based on the function of time but influenced additively by velocity, and the model (4) is the same as the model (3) but are influenced by velocity multiplicatively. Although it is very difficult to identify the exact form of the function $F(T)$ and $G(V)$, there are a few candidates for the form from the past researches. As for $F(T)$, the previous psychophysical studies of duration estmation have suggested that the perceived duration is a linear function or a power function of the stimulus duration (Macar, 1985). In the following analysis, the next linear function was only used, because of its simplicity in multiple regression analysis and the slightness in the deviation of the obtained exponents for the power function from the unity.

$$
\begin{aligned}
& F(T)=k_{1} T+k_{2} \\
& \left(k_{1}, k_{2}=\text { constant }\right)
\end{aligned}
$$

As for $G(V)$, the function which represents the influence of stimulus velocity upon estimated duration seems to have a quadratic form from Table 1. Thus the linear and quadratic function were used as the candidates. But it should not be necessarily considered that the estimated values are influenced directly by the raw 
magnitude of velocity. Tentatively we uscd the raw magnitude and the logarithmically transformed values. Thus the next four functions were derived.

$$
\begin{aligned}
& G(V)=k_{1} V+k_{2} \\
& G(V)=k_{1} V^{2}+k_{2} V+k_{3} \\
& G(V)=k_{1} \log V+k_{2} \\
& G(V)=k_{1}(\log V)^{2}+k_{2} \log V+k_{3} \\
& \left(k_{1}, k_{2}, k_{3}=\text { constant }\right)
\end{aligned}
$$

By replacing $F(T), G(V)$ in (3) and (4) with their equivalent functions (5)-(9), eight possible combination models can be built up. Those are presented in the left column of Table 2.

To choose the best model among them, the multiple regression analysis was performed. ${ }^{2}$ The response variable of each model was the estimated value $E$, which is given by multiplying EOS by sd (or pd) in each condition, and the regressor variables were the stimulus duration (or produced duration) $T$, and the stimulus velocity $V$ or $\log V$, and their multiplicative combinations. The estimates of parameters, the RSQs (the squared multiple correlation coefficients) and AICs (Akaike's information criterions (Akaike, 1969)) in each model were presented in Table 2. From the point of view of RSQs and AICs, the best model among eight models was the model (8) shown in Table 2 in both experiments. This model is not only a multiplicative one like formula (4) but also contains a quadratic function of stimulus velocity logarithmically transformed. This fact is quite important. Because, unless we had chosen the quadratic function of velocity or chosen the logarithmically transformation, we would not have obtained such a good agreement. We examined other simpler models containing parameters whose number is less than four but failed to find such an agreement.

2 In this analysis, the SAS software procedures, REG, RSQUARE and NLIN were used.
Still more we analyzed Tayama and Aiba's (1982) data in the same way and also found that the model (8) was the best one. The fact that irrelevant to the difference of the experimental methods the model (8) was the best model has an important implication about the complicated way the velocity influences upon time estimation.

\section{General Discussion}

In the present study we used a rotating-spot-pattern. In such a stimulus the number of spots was always constant and we could disregard the possibility of the frequency effect which might have been present with the random-spot-pattern moving horizontally which Tayama and Aiba (1982) had used. Despite this obvious difference, we found substantially the same results as those of Tayama and Aiba (1982). In both studies, two effects were found; the higher was the velocity for a moving pattern, the larger was the estimate, and the estimate for stationary pattern was not necessarily smaller than that for moving pattern.

Moreover, we used and compared two methods of time estimation, i.e. magnitude estimation vs. magnitude production. There were no difference between the two methods with respect to already mentioned two effects, particularly with respect to the mean EOS's. This suggests that the two effects are by no means artifacts of a particular experimental method like verbal estimation methods. The difference of the two methods were reflected, if any, in the absolute exponent values (the slopes in Fig. 4) and in the parameter estimates of the models in multiple regression analysis.

The exponent analysis has shown that its value increased with the decrease of velocity for the pattern, irrespective of whether the pattern was moving or stationary. With respect to this point we obtained a consistent result in both 
methods. This fact suggest that the durations for the higher velocity pattern were estimated relatively longer in the range of short durations and relatively shorter in the range of long durations. But the mean estimates for the higher velocity pattern were relatively larger than those for the lower velocity pattern irrelevant to the length of the duration. Therefore these imply that the velocity of the pattern had a strong influence upon time estimation, especially in the range of short durations.

Here let us consider the next three problems: (i) Why the velocity effect have occurred? (ii) Why the estimate for stationary pattern was not the smallest? (iii) Whether the two effects are based on one and the same process or not? These are not so different from one another in essence. But at first, consider the question (i).

If we deal only with the velocity effect upon duration estimation, we can simply think of two possibilities for the effect. The one possibility concerns the duration of visible persistence for movement. Recently, Farrell (1984) suggested that the visual persistence increases with the distance separating that stimulus from other stimuli. If we substitute velocity for distance and apply this suggestion to our problem, the velocity effect will be explained well because the higher is the velocity, the longer will be the duration of the visible persistence, and naturally the larger will be the estimates. But Farrell's suggestion only refers to a very briefly presented stimulus, less than 250 ms. Still more, the substitution of velocity for distance may not be justifiable.

The second possibility concerns higher order cognitive processes. Developmental researchers (Cohen, 1967; Friedman, 1982; Piaget, 1969, 1981, etc.) have shown that the children's estimates of time are readily affected by velocity or distance. Piaget (1981) said that, for adults, perception of time is based on the same law as that which governs children's perception. According to him a more rapid movement is judged shorter in time when the subject's attention is drawn to velocity. Piaget's result was opposite to ours but his view suggested that the velocity effect might depend upon the empirical cognitive process, the subject's knowledge or concept of time in which faster may equal less time as Piaget found, or indeed more time as the present results indicate.

But if one of these two possibilities is correct, a contradiction will arise with respect to the question (ii). These possibilities predict clearly that the duration for the stationary pattern would be necessarily estimated shorter than that for moving pattern, but in the present experiments, we found the contrary. But this interpretation is applied to the case that the two effects are based on one and the same process. Thus next we can not help taking account of the question (iii). It is very difficult to say clearly that the two effects are not based on one and the same process. Because we can roughly say that it is one and the same process of time estimation as a whole even though the process has many different subprocessors. At least we had better not consider that the unidimensional model (Kowal, 1981) can explain the two effects.

We analyzed our experimental data according to the models which have two sub-processors like model (3) or (4) by multiple regression analysis and decided on a best model. The best model was not an additive one like the Thomas and Weaver (1975) model but a multiplicative one and did not contain a simple unidimensional function of velocity but contained a quadratic function of logarithmically transformed velocity. Although it is a rather complicated one, only such a model can explain the salient influence of velocity upon time estimation, disregarding for the moment the differ- 
ence of methods. Pcrhaps, there might be other complex mathematical models which can better explain our experimental results. For cxample, if we choose the power function as the basic time function and the exponent is an appropriate one, the parameter estimates of the model will produce better fit to experimental results, or if the exponent is the function of velocity, we might be able to explain even the exponent results.

We should keep in mind that we might have neglected very important cognitive factors which should be incorporated into the regression models, for instance, factors of attention (Tayama \& Aiba, 1982; Thomas \& Weaver, 1975; Underwood, 1975), temporal context (Block, 1978, 1979), expectation, attitude, etc. Tayama and Aiba (1982) assumed that the two effects are based on two different processes. Namely cxposed to higher velocity stimuli the subject's internal schema is modified rapidly and there occurs the velocity effect proportional to the rapidity (estimated duration increases with velocity). On the other hand, the subject's attention toward passed time is strengthened with the stationarity for its uniqueness against all other moving stimuli and this is the reason why the estimates for the stationary pattern was not necessarily smaller than that for moving pattern. At the present point we are basically in almost the same position as Tayama and Aiba's. But we might also add that the attention to passed time could be augmented in the stationary pattern by its sheer lack of any changes especially in longer duration. This is supported by our exponent analysis.

In order to develop a sophisticated model to explain the influence of velocity upon time estimation completely, we have to examine the velocity effects in more detail, especially in very slow velocity conditions, and still more, in conjunction with various methods. Also we might need to investigate these ef- fects from various points of view. Further experimental researches are required all the more because what little we discovered in this study promises a development of a more general model or principle in time estimation.

\section{References}

Abbe, S. 1935 Experimental study on the correlation between time and space. Tohoku Psychologia Folia, 13, 53-68.

Akaike, H. 1969 Fitting autoregressive models for prediction. Annals of the Institute of Statistical Mathematics, 21, 243-247.

Avant, L. L., Lyman, P. J., \& Antes, J. R. 1975 Effects of stimulus familiarity upon judged visual duration. Perception \& Psychophysics, 17, 253262.

Block, R. A. 1978 Remembered duration: Effects of event and sequence complexity. Memory and Cognition, 6, 320-326.

Block, R. A. 1979 Time and consciousness. In G. Underwood \& R. Stevens (Eds.), Aspects of consciousness. Vol. 1. London: Academic Press. Pp. 179-217.

Cahen, J. 1967 Psychological time in health and disease. Springfield, Ill: Charles C. Thomas.

Cohen, J., Hunsel, C.E.M., \& Sylvester, J. D. 1955 Interdependence in judgments of space, time, and movement. Acta Psychologia, 1, 360372.

Farrell, S. E. 1984 Visible persistence of moving objects. Journal of Experimental Psychology: Human Perception and Performance, 10, 502-511.

Fraisse, P. 1962 Influence de la vitess des mouvements sur l'estimation de leur duree. Annee Psychologique, 62, 391-399.

Fraisse, P. 1963 The psychology of time. New York: Harper \& Row.

Friedman, W.J. (Ed.) 1982 The developmental psychology of time. New York: Academic Press.

Kowal, K.H. 1981 Growth of apparent duration: Effect of melodic and non-melodic tonal variation. Perceptual and Molor Skills, 52, 803817.

Lhamon, W., \& Goldstone, S. 1975 Movement and the judged duration of visual targets. Bulletin of the Psychonomic Society, 5, 53-54.

Macar, F. 1985 Time psychophysics and related models. In J. A. Michon \& J. L. Jackson (Eds), Time, mind, and behavior. Berlin: Springer-Verlag. Pp. 112-130. 
Matsuda, F. 1974 Effects of space and velocity on time estimation in children and adults. Japanese Psychological Rescarch, 37, 107-123.

Mo, S. S. 1971 Judgment of temporal duration as a function of numerosity. Psychonomic Sciene, 24, $71-72$.

Ornstein, R. E. 1969 On the expericuce of time. Harmondsworth: Penguin Books.

Piaget, J. 1969 The Child's conception of time. (Trs. by A.J. Pomerans) London: Routledge \& Kegan Paul. (Le developpement de la notion de temps che l'enfant. 1946 Paris: Presses Universitaires de France.)

Piaget, J. 1981 Time perception in children. In J.T. Fraser (Ed.), The voites of time. 2nd ed. Amherst: University of Massachusetts Press. Pp. 202-216.

Rachlin, H. C. 1966 Scaling subjective velocity, distance, and duration. Perception $\mathcal{S}^{2}$ Psychophysics, 1, 77-82.

Tayama, T. 1984 Global matching and local matching in perceiving velocity changes. Japanese Joumal of Psychology, 55, 275-281. (In Japa- nese with English abstract)

Tayama, T. 1986 The diminution of spatial effects upon duration estimation with focused attention. Japanese Journal of Psychology, 57, 9599. (In Japanese with English abstract)

Tayama, T., \& Aiba, T.S. 1982 The influence of spatial attributes of stimuli upon time estimation. Hokkaido Behavioral Report Series P, Supplement. No. 28. (In Japanese with English abstract)

Thomas, E.A.C., \& Brown, I., Jr. 1974 Time perception and the filled-duration illusion. Perception \& Psychophysics, 16, 449-458.

Thomas, E.A.C., \& Cantor, N.E. 1976 Simultaneous time and size perception. Perception $\mathcal{E}^{\circ}$ Psychophysics, 19, 353-360.

Thomas, E.A.C., \& Weaver, W. B. 1975 Cognitive processing and time perception. Perception \& Psychophysics, 17, 363-367.

Underwood, G, 1975 Attention and the perception of duration during encoding and retrieval. Perception, 2, 291-296.

(Received Sept. 1, 1986; accepted July 11, 1987) 